\title{
Spectra of locally matrix algebras
}

\author{
O. Bezushchak
}

Communicated by A. P. Petravchuk

\begin{abstract}
A BStRACT. We describe spectra of associative (not necessarily unital and not necessarily countable-dimensional) locally matrix algebras. We determine all possible spectra of locally matrix algebras and give a new proof of Dixmier-Baranov Theorem. As an application of our description of spectra, we determine embeddings of locally matrix algebras.
\end{abstract}

\section{Introduction}

Let $\mathbb{F}$ be a ground field. Recall that an associative $\mathbb{F}$-algebra $A$ is called a locally matrix algebra (see [10]) if for an arbitrary finite subset of $A$ there exists a subalgebra $B \subset A$ containing this subset and such that $B$ is isomorphic to a matrix algebra $M_{n}(\mathbb{F})$ for some $n \geqslant 1$. In what follows we will sometimes identify $B$ and $M_{n}(\mathbb{F})$, that is, assume that $M_{n}(\mathbb{F}) \subset A$. We call a locally matrix algebra unital if it contains 1 .

Let $A$ be a countable-dimensional unital locally matrix algebra. In [7], J.G. Glimm defined the Steinitz number st $(A)$ of the algebra $A$ and proved that $A$ is uniquely determined by $\operatorname{st}(A)$. J. Dixmier [5] showed that non-unital countable-dimensional locally matrix algebras over the field of complex numbers can be parameterized by pairs $(s, \alpha)$, where $s$ is a Steinitz number and $\alpha$ is a nonnegative real number. A.A. Baranov [1] extended this parametrization to locally matrix algebras over arbitrary fields.

2020 MSC: 08A05, 16S50.

Key words and phrases: locally matrix algebra, Steinitz number, spectrum, embedding. 
In [2], we defined the Steinitz number st $(A)$ for a unital locally matrix algebra $A$ of an arbitrary dimension. We showed that for a unital locally matrix algebra $A$ of dimension $>\aleph_{0}$ the Steinitz number $\operatorname{st}(A)$ no longer determines $A$; see $[3,4]$. However, it determines the universal elementary theory of $A[3]$.

In this paper for an arbitrary (not necessarily unital and not necessarily countable-dimensional) locally matrix algebra $A$, we define a subset of $\mathbb{S N}$ that we call the spectrum of $A$ and denote as $\operatorname{Spec}(A)$. We determine all possible spectra of locally matrix algebras and give a new proof of Dixmier-Baranov Theorem. As an application of our description of spectra, we determine embeddings of locally matrix algebras.

\section{Spectra of locally matrix algebras}

Let $\mathbb{P}$ be the set of all primes and $\mathbb{N}$ be the set of all positive integers. A Steinitz number (see [11]) is an infinite formal product of the form

$$
\prod_{p \in \mathbb{P}} p^{r_{p}}
$$

where $r_{p} \in \mathbb{N} \cup\{0, \infty\}$ for all $p \in \mathbb{P}$.

Denote by $\mathbb{S N}$ the set of all Steinitz numbers. Notice, that the set of all positive integers $\mathbb{N}$ is a subset of $\mathbb{S N}$. The numbers $\mathbb{S N} \backslash \mathbb{N}$ are called infinite Steinitz numbers.

Let $A$ be a locally matrix algebra with a unit 1 over a field $F$ and let $D(A)$ be the set of all positive integers $n$ such that there is a subalgebra $A^{\prime}, 1 \in A^{\prime} \subseteq A, A^{\prime} \cong M_{n}(F)$. Then the least common multiple of the set $D(A)$ is called the Steinitz number of the algebra $A$ and denoted as $\mathbf{s t}(A)$; see [2].

Now let $A$ be a (not necessarily unital) locally matrix algebra. For an arbitrary idempotent $0 \neq e \in A$ the subalgebra $e A e$ is a unital locally matrix algebra. That is why we can talk about its Steinitz number st $(e A e)$. The subset

$$
\operatorname{Spec}(A)=\left\{\operatorname{st}(e A e) \mid e \in A, e \neq 0, e^{2}=e\right\},
$$

where $e$ runs through all nonzero idempotents of the algebra $A$, is called the spectrum of the algebra $A$.

For a Steinitz number $s$ let $\Omega(s)$ denote the set of all natural numbers $n \in \mathbb{N}$ that divide $s$.

For a Steinitz numbers $s_{1}, s_{2}$ we say that $s_{1}$ finitely divides $s_{2}$ if there exists $b \in \Omega\left(s_{2}\right)$ such that $s_{1}=s_{2} / b$ (we denote: $\left.s_{1}\right|_{\text {fin }} s_{2}$ ). 
Steinitz numbers $s_{1}, s_{2}$ are rationally connected if $s_{2}=q \cdot s_{1}$, where $q$ is some rational number.

We call a subset $S \subset \mathbb{S N}$ saturated if

1) any two Steinitz numbers from $S$ are rationally connected;

2) if $s_{2} \in S$ and $\left.s_{1}\right|_{\text {fin }} s_{2}$ then $s_{1} \in S$;

3) if $s, n s \in S$, where $n \in \mathbb{N}$, then $i s \in S$ for any $i, 1 \leqslant i \leqslant n$.

Theorem 1. For an arbitrary locally matrix algebra $A$ its spectrum is a saturated subset of $\mathbb{S N}$.

Let us consider examples of saturated subsets of $\mathbb{S N}$.

Example 1. For an arbitrary natural number $n$ the set $\{1,2, \ldots, n\}$ is saturated.

Example 2. Let $s$ be a Steinitz number. The set

$$
S(\infty, s):=\left\{\frac{a}{b} \cdot s \mid a \in \mathbb{N}, b \in \Omega(s)\right\}
$$

is saturated. For an arbitrary Steinitz number $s^{\prime} \in S(\infty, s)$ we have $S(\infty, s)=S\left(\infty, s^{\prime}\right)$. If $s \in \mathbb{N}$ then $S(\infty, s)=\mathbb{N}$.

Example 3. Let $r$ be a real number, $1 \leqslant r<\infty$. Let $s$ be an infinite Steinitz number. The set

$$
S(r, s)=\left\{\frac{a}{b} s \mid a, b \in \mathbb{N} ; b \in \Omega(s), a \leqslant r b\right\}
$$

is saturated.

Example 4. Let $s$ be an infinite Steinitz number and let $r=u / v$ be a rational number; $u, v \in \mathbb{N}, v \in \Omega(s)$. Then the set

$$
S^{+}(r, s)=\left\{\frac{a}{b} s \mid a, b \in \mathbb{N} ; b \in \Omega(s), a<r b\right\}
$$

is saturated.

Theorem 2. Every saturated subset of $\mathbb{S N}$ is one of the following sets:

1) $\{1,2, \ldots, n\}, n \in \mathbb{N}$, or $\mathbb{N}$;

2) $S(\infty, s), s \in \mathbb{S} \backslash \mathbb{N}$;

3) $S(r, s)$, where $s \in \mathbb{S N} \backslash \mathbb{N}, r \in[1, \infty)$;

4) $S^{+}(r, s)$, where $s \in \mathbb{S} \backslash \mathbb{N}, r=u / v, u \in \mathbb{N}, v \in \Omega(s)$.

Remark 1. The real number $r$ above is the inverse of the density invariant of Dixmier-Baranov. 
Theorem 3. (1) For any saturated subset $S \subseteq \mathbb{S N}$ there exists a countable-dimensional locally matrix algebra $A$ such that $\operatorname{Spec}(A)=S$.

(2) If $A, B$ are countable-dimensional locally matrix algebras and $\operatorname{Spec}(A)=\operatorname{Spec}(B)$ then $A \cong B$.

Remark 2. The part (2) of Theorem 3 is a new proof of Dixmier-Baranov Theorem.

Which spectra above correspond to unital algebras?

Theorem 4. A locally matrix algebra $A$ is unital if and only if $\operatorname{Spec}(A)=$ $\{1,2, \ldots, n\}$, where $n \in \mathbb{N}$, or $\operatorname{Spec}(A)=S(r, s)$, where $s \in \mathbb{S N} \backslash \mathbb{N}$, $r=u / v, u, v \in \mathbb{N}, v \in \Omega(s)$.

Proof of Theorem 1. In what follows, we assume that $A$ is a locally matrix $\mathbb{F}$-algebra. Recall the partial order on the set of all idempotents of $A$ : for idempotents $e, f \in A$ we define $e \geqslant f$ if $f \in e A e$.

We claim that for arbitrary idempotents $e_{1}, e_{2} \in A$ there exists an idempotent $e_{3} \in A$ such that $e_{1} \leqslant e_{3}, e_{2} \leqslant e_{3}$. Indeed, there exists a subalgebra $A^{\prime} \subset A$ such that $e_{1}, e_{2} \in A^{\prime}$ and $A^{\prime} \cong M_{n}(\mathbb{F}), n \geqslant 1$. Let $e_{3}$ be the identity element of the subalgebra $A^{\prime}$. Then $e_{1} \leqslant e_{3}, e_{2} \leqslant e_{3}$.

Now suppose that the locally matrix algebra $A$ is unital. Let $a \in A$. Choose a subalgebra $A^{\prime} \subset A$ such that $1 \in A^{\prime}, a \in A^{\prime}$ and $A^{\prime} \cong M_{n}(\mathbb{F})$, $n \geqslant 1$. Let $r$ be the range of the matrix $a$ in $A^{\prime}$. Let

$$
r(a)=\frac{r}{n}, \quad 0 \leqslant r(a) \leqslant 1 .
$$

V.M. Kurochkin [9] noticed that the number $r(a)$ does not depend on a choice of a subalgebra $A^{\prime}$. We call $r(a)$ the relative range of the element $a$. In [4], we showed that if $A$ is a unital locally matrix algebra and $e \in A$ is an idempotent, then $\mathbf{s t}(e A e)=r(e) \cdot \mathbf{s t}(A)$.

Now let $A$ be a not necessarily unital locally matrix algebra. Let $e_{1}$, $e_{2} \in A$ be idempotents. Choose an idempotent $e_{3} \in A$ such that $e_{1} \leqslant e_{3}$, $e_{2} \leqslant e_{3}$, i.e. $e_{1}, e_{2} \in e_{3} A e_{3}$. Let $q_{1}, q_{2}$ be relative ranges of the idempotents $e_{1}, e_{2}$ in the unital locally matrix algebra $e_{3} A e_{3}$. Then

$$
\mathbf{s t}\left(e_{1} A e_{1}\right)=q_{1} \mathbf{s t}\left(e_{3} A e_{3}\right), \quad \boldsymbol{s t}\left(e_{2} A e_{2}\right)=q_{2} \mathbf{s t}\left(e_{3} A e_{3}\right) .
$$

This implies that the Steinitz numbers $\mathbf{s t}\left(e_{1} A e_{1}\right), \mathbf{s t}\left(e_{2} A e_{2}\right)$ are rationally connected. We have checked the condition 1 ) from the definition of saturated sets. 
Let $0 \neq e \in A$ be an idempotent. Let $s_{2}=\mathbf{s t}(e A e), k \in \Omega\left(s_{2}\right)$ and let $s_{1}=s_{2} / k$. The unital locally matrix algebra $e A e$ contains a subalgebra $e \in M_{k}(\mathbb{F}) \subset e A e$. Consider the matrix unit $e_{11}$ of the algebra $M_{k}(\mathbb{F})$. The relative range of the idempotent $e_{11}$ in the unital algebra $e A e$ is equal to $1 / k$. Hence

$$
\mathbf{s t}\left(e_{11} A e_{11}\right)=\frac{1}{k} \mathbf{s t}(e A e)=s_{1}, \quad s_{1} \in \operatorname{Spec}(A) .
$$

We have checked the condition 2).

Now let $n \geqslant 1$. Suppose that Steinitz numbers $s$ and $n s$ lie in $\operatorname{Spec}(A)$. It means that there exist idempotents $e_{1}, e_{2} \in A$ such that $s=\operatorname{st}\left(e_{1} A e_{1}\right)$, $n s=\mathbf{s t}\left(e_{2} A e_{2}\right)$. There exists a matrix subalgebra $M_{k}(\mathbb{F}) \subset A$ that contains $e_{1}$ and $e_{2}$. As above, let $e_{3}$ be the identity element of the algebra $M_{k}(\mathbb{F})$. Let $\mathrm{rk}\left(e_{i}\right)$ be the range of the idempotent $e_{i}$ in the matrix algebra $M_{k}(\mathbb{F})$. We have

$$
s=\frac{\mathrm{rk}\left(e_{1}\right)}{k} \cdot \mathbf{s t}\left(e_{3} A e_{3}\right), \quad n s=\frac{\mathrm{rk}\left(e_{2}\right)}{k} \cdot \mathbf{s t}\left(e_{3} A e_{3}\right)
$$

which implies $\operatorname{rk}\left(e_{2}\right)=n \cdot \operatorname{rk}\left(e_{1}\right)$. In particular, $n \cdot \operatorname{rk}\left(e_{1}\right) \leqslant k$. Let $1 \leqslant i \leqslant n$. Consider the idempotent

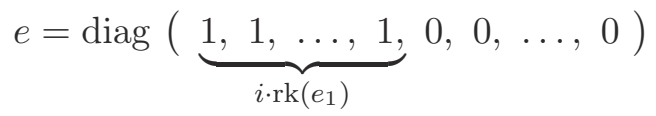

in the matrix algebra $M_{k}(\mathbb{F})$. We have

$$
\mathbf{s t}(e A e)=\frac{i \cdot \operatorname{rk}\left(e_{1}\right)}{k} \cdot \mathbf{s t}\left(e_{3} A e_{3}\right)=i \cdot \mathbf{s t}\left(e_{1} A e_{1}\right)=i s .
$$

We showed that is $\in \operatorname{Spec}(A)$. Hence $\operatorname{Spec}(A)$ is a saturated subset of $\mathbb{S N}$. It completes the proof of Theorem 1.

\section{Classification of saturated subsets of $\mathbb{S N}$}

Our aim in this section is to classify all saturated subsets of $\mathbb{S} \mathbb{N}$. We remark that if at least one Steinitz number from a saturated set $S$ is infinite then by the condition 1) all Steinitz numbers from $S$ are infinite.

Let $S$ be a saturated subset of $\mathbb{S N}$. For a Steinitz number $s \in S$ and for a natural number $b \in \Omega(s)$ let

$$
r_{s}(b)=\max \left\{i \geqslant 1 \mid i \cdot \frac{s}{b} \in S\right\} \text {. }
$$


Lemma 1. If there exists a Steinitz number $s_{0} \in S$ and a natural number $b_{0} \in \Omega\left(s_{0}\right)$ such that $r_{s_{0}}\left(b_{0}\right)=\infty$ then for any $s \in S$ and any $b \in \Omega(s)$ we have $r_{s}(b)=\infty$.

Proof. Let us show at first that $r_{s_{0}}(b)=\infty$ for any $b \in \Omega\left(s_{0}\right)$. Indeed, there exists a natural number $c \in \Omega\left(s_{0}\right)$ such that both $b_{0}$ and $b$ divide $c$. Then for an arbitrary $i \geqslant 1$ we have

$$
i \cdot \frac{s_{0}}{b_{0}}=\left(i \cdot \frac{c}{b_{0}}\right) \cdot \frac{s_{0}}{c} \in S .
$$

This implies that $r_{s_{0}}(c)=\infty$. Hence,

$$
i \cdot \frac{s_{0}}{b}=\left(i \cdot \frac{c}{b}\right) \cdot \frac{s_{0}}{c} \in S,
$$

which proves the claim.

Now choose an arbitrary Steinitz number $s \in S$. By the condition 1), the Steinitz numbers $s$ and $s_{0}$ are rationally connected, i.e. there exist $a \in \mathbb{N}, b \in \Omega\left(s_{0}\right)$ such that $s=(a / b) \cdot s_{0}$. By the condition 2$), s_{0} / b \in S$. Choose a natural number $c \in \Omega\left(s_{0} / b\right)$. Then $c \in \Omega(s)$ and $b c \in \Omega\left(s_{0}\right)$. For an arbitrary $i \geqslant 1$ we have $i \cdot s / c=i \cdot a \cdot s_{0} /(b c) \in S$ since $r_{s_{0}}(b c)=\infty$. This implies $r_{s}(c)=\infty$ and completes the proof of the lemma.

If a saturated set satisfies the assumptions of Lemma 1 then it is referred to as a set of infinite type. Otherwise, we talk about a saturated set of finite type.

Lemma 2. 1) For an arbitrary Steinitz number $s_{0} \in \mathbb{S N}$ the set

$$
S\left(\infty, s_{0}\right):=\left\{\frac{a}{b} \cdot s_{0} \mid a \in \mathbb{N}, b \in \Omega\left(s_{0}\right)\right\}
$$

is a saturated set of infinite type.

2) If $S$ is a saturated set of infinite type, then for an arbitrary Steinitz number $s \in S$ we have $S=S(\infty, s)$.

Proof. We have to show that the set $S\left(\infty, s_{0}\right)$ satisfies the conditions 1$)$, $2), 3)$. The condition 1$)$ is obvious. Let $s=(a / b) \cdot s_{0}, b \in \Omega\left(s_{0}\right)$. Without loss of generality, we assume that $a$ and $b$ are coprime. Let $c \in \Omega(s)$ and let $d=\operatorname{gcd}(c, a)$ be the greatest common divisor of $a$ and $c, a=a^{\prime} d$, $c=c^{\prime} d$, the numbers $a^{\prime}, c^{\prime}$ are coprime. Then $a \cdot s_{0} /(b c)=a^{\prime} \cdot s_{0} /\left(b c^{\prime}\right)$, which implies that $d c^{\prime} \in \Omega\left(s_{0}\right)$. Hence

$$
\frac{s}{c}=\frac{a}{b c} \cdot s_{0}=\frac{a^{\prime}}{b c^{\prime}} \cdot s_{0} \in S\left(\infty, s_{0}\right) .
$$


We have checked the condition 2).

Let us check the condition 3). Choose $s=(a / b) \cdot s_{0} \in S\left(\infty, s_{0}\right)$, $b \in \Omega\left(s_{0}\right)$. Let $c \in \Omega(s)$. We need to check that for any $i \geqslant 1$

$$
i \cdot \frac{s}{c}=\frac{i a}{b c} \cdot s_{0} \in S\left(\infty, s_{0}\right) \text {. }
$$

Let $a /(b c)=a^{\prime} / b^{\prime}$, where the natural numbers $a^{\prime}, b^{\prime}$ are coprime. Since

$$
\frac{a}{b c} \cdot s_{0}=\frac{s}{c} \in \mathbb{S N}
$$

it follows that $b^{\prime} \in \Omega\left(s_{0}\right)$. Hence, $i \cdot\left(a^{\prime} / b^{\prime}\right) \cdot s_{0} \in S\left(\infty, s_{0}\right)$, which implies that $S\left(\infty, s_{0}\right)$ satisfies the condition 3$)$ and, therefore, is saturated.

Let $S$ be a saturated subset of $\mathbb{S N}$ of infinite type. Choose $s_{0} \in S$. Our aim is to show that $S=S\left(\infty, s_{0}\right)$. Since the subset $S$ is of infinite type it follows that $r_{s}(b)=\infty$ for any $s \in S, b \in \Omega(s)$. In particular,

$$
S\left(\infty, s_{0}\right)=\left\{\frac{a}{b} \cdot s_{0} \mid s \in \Omega\left(s_{0}\right)\right\} \subseteq S .
$$

An arbitrary Steinitz number $s \in S$ is rationally connected to $s_{0}$, hence there exist $a, b \in \mathbb{N}$ such that $s=(a / b) \cdot s_{0}$. Without loss of generality, we assume that $a$ and $b$ are coprime, which implies $b \in \Omega\left(s_{0}\right)$. We proved that $s \in S\left(\infty, s_{0}\right)$.

Now let $S \subset \mathbb{S N}$ be a saturated subset of finite type, that is, for any $s \in S, d \in \Omega(s)$ we have

$$
r_{s}(b)=\max \left\{i \in \mathbb{N} \mid i \cdot \frac{s}{b} \in S\right\}<\infty .
$$

By the condition 3),

$$
\left\{i \in \mathbb{N} \mid i \cdot \frac{s}{b} \in S\right\}=\left[1, r_{s}(b)\right] .
$$

Since $b \cdot(s / b) \in S$ it follows that $b \leqslant r_{s}(b)$. Choose a Steinitz number $s \in S$ and two natural numbers $b, c \in \Omega(s)$ such that $b$ divides $c$. If $i \cdot(s / b) \in S$ then $(i c / b) \cdot(s / c) \in S$. Hence $r_{s}(b) \cdot(c / b) \leqslant r_{s}(c)$. In other words,

$$
\frac{r_{s}(b)}{b} \leqslant \frac{r_{s}(c)}{c} .
$$

Let $i \in \mathbb{N}, s / c \in S$ and let $k$ be a maximal nonnegative integer such that $k \cdot(c / b) \leqslant i$. By the condition 3$), k \cdot(c / b) \cdot(s / c) \in S$, hence $k \cdot(s / b) \in S$. So, $k \leqslant r_{s}(b)$. We proved that

$$
\left[\frac{r_{s}(c)}{c / b}\right] \leqslant r_{s}(b) .
$$


The inequalities (1), (2) imply

$$
\left[\frac{r_{s}(c)}{c / b}\right] \leqslant r_{s}(b) \leqslant \frac{r_{s}(c)}{c / b} .
$$

Hence

$$
r_{s}(b)=\left[\frac{r_{s}(c)}{c / b}\right]
$$

In particular,

$$
\frac{r_{s}(c)}{c / b}-1<r_{s}(b), \quad \frac{r_{s}(c)}{c / b}<r_{s}(b)+1 .
$$

Dividing by $b$, we get

$$
\frac{r_{s}(b)}{b} \leqslant \frac{r_{s}(c)}{c}<\frac{r_{s}(b)}{b}+\frac{1}{b} .
$$

Lemma 3. Let $S \subset \mathbb{S N}$ be a saturated subset of finite type and let $s \in S$ be an infinite Steinitz number. Then there exists a limit

$$
r_{S}(s)=\lim _{\substack{b \in \Omega(s) \\ b \rightarrow \infty}} \frac{r_{s}(b)}{b}, \quad 1 \leqslant r_{S}(s)<\infty .
$$

If the set $S$ is fixed then we denote $r_{S}(s)=r(s)$.

Remark 3. The limit $r(s)$ is equal to the inverse of the density invariant of Dixmier-Baranov $[1,5]$.

The proof of Lemma 3. The set $\left\{r_{s}(b) / b \mid b \in \Omega(s)\right\}$ is bounded from above. Indeed, choose $b_{0} \in \Omega(s)$. For an arbitrary $b \in \Omega(s)$ there exists $c \in \Omega(s)$ that is a common multiple for $b_{0}$ and $b$. Then by (1) and (4),

$$
\frac{r(b)}{b} \leqslant \frac{r(c)}{c}<\frac{r\left(b_{0}\right)}{b_{0}}+\frac{1}{b_{0}} .
$$

Let

$$
r=r(s)=\sup \left\{\frac{r_{s}(b)}{b} \mid b \in \Omega(s)\right\} .
$$

Clearly, $1 \leqslant r<\infty$. Choose $\varepsilon>0$. Let $N(\varepsilon)=[2 r / \varepsilon]+1$. We will show that for any $b \in \Omega(s), b \geqslant N(\varepsilon)$, we have $r-\varepsilon<r_{s}(b) / b$.

Indeed, let $b \in \Omega(s), b \geqslant N(\varepsilon)>2 r / \varepsilon$. Then $1 / b<\varepsilon /(2 r) \leqslant \varepsilon / 2$. There exists a natural number $b_{0} \in \Omega(s)$ such that $r-\varepsilon / 2<r_{s}\left(b_{0}\right) / b_{0}$. Let $c \in \Omega(s)$ be a common multiple of $b_{0}$ and $b$. Then (4) implies

$$
\frac{r(b)}{b}>\frac{r(c)}{c}-\frac{1}{b} \geqslant \frac{r\left(b_{0}\right)}{b_{0}}-\frac{1}{b}>r-\frac{\varepsilon}{2}-\frac{\varepsilon}{2}=r-\varepsilon .
$$


So,

$$
r=\lim _{\substack{b \in \Omega(s) \\ b \rightarrow \infty}} \frac{r_{s}(b)}{b}
$$

and this completes the proof of the lemma.

Lemma 4. Let $s, s^{\prime} \in S$ be infinite Steinitz numbers, $s^{\prime}=(a / b) \cdot s ; a$, $b \in \mathbb{N} ; b \in \Omega(s)$. Then $r\left(s^{\prime}\right)=(a / b) \cdot r(s)$.

Proof. It is sufficient to show that if $s, m s \in S, m \in \mathbb{N}$, then $m \cdot r(m s)=$ $r(s)$.

Suppose that $b \in \Omega(s)$ and $i \cdot(m s / b) \in S$. Then $i \cdot m \cdot(s / b) \in S$. Hence $r_{m s}(b) \cdot m \leqslant r_{s}(b)$ and, therefore, $r(m s) \cdot m \leqslant r(s)$.

On the other hand, if $i \cdot(s / b) \in S$ then $[i / m] \cdot m \leqslant i$ and, therefore, $[i / m] \cdot m \cdot(s / b) \in S$. We showed that

$$
\begin{gathered}
{\left[\frac{r_{s}(b)}{m}\right] \leqslant r_{m s}(b), \quad \frac{r_{s}(b)}{m}-1<r_{m s}(b),} \\
\frac{1}{m} \cdot \frac{r_{s}(b)}{b}-\frac{1}{b}<\frac{r_{m s}(b)}{b} .
\end{gathered}
$$

Assuming $b \rightarrow \infty$ we get $(1 / m) \cdot r(s) \leqslant r(m s)$, which completes the proof of the lemma.

In the inequality (4), let $c \rightarrow \infty$. Then

$$
\frac{r_{s}(b)}{b} \leqslant r(s) \leqslant \frac{r_{s}(b)}{b}+\frac{1}{b}, \quad r_{s}(b) \leqslant r(s) b \leqslant r_{s}(b)+1 .
$$

If the number $r(s)$ is irrational then $r_{s}(b)=[r(s) b]$ for all $b \in \Omega(s)$.

Now suppose that the number $r=r_{s}(b)$ is rational; $r=u / v ; u, v$ are coprime. If a number $b \in \Omega(s)$ is not a multiple of $v$ then, as above, $r_{s}(b)=[(u / v) \cdot b]$. If $b$ is a multiple of $v$ then

$$
r_{s}(b)=\left[\begin{array}{llll}
r & b & & \text { or } \\
r & b & -1
\end{array}\right.
$$

Lemma 5. If at least for one number $b_{0} \in \Omega(s) \bigcap v \mathbb{N}$ we have $r_{s}\left(b_{0}\right)=r b_{0}$ then for all $b \in \Omega(s) \bigcap v \mathbb{N}$ we have $r_{s}(b)=r b$.

Proof. Let $b, c \in \Omega(s) \bigcap v \mathbb{N}$ and $b$ divides $c$. If $r_{s}(b)=r b$ then, by the inequality (1), we have

$$
r=\frac{r_{s}(b)}{b} \leqslant \frac{r_{s}(c)}{c}
$$


which implies $r_{s}(c)=r c$. On the other hand, if $r_{s}(c)=r c$ then, by the inequality (4),

$$
r=\frac{r_{s}(c)}{c}<\frac{r_{s}(b)}{b}+\frac{1}{b},
$$

which implies $r_{s}(b)>r b-1$. Hence $r_{s}(b)=r b$. We showed that $r_{s}(b)=r b$ if and only if $r_{s}(c)=r c$.

Now choose $b_{1}, b_{2} \in \Omega(s) \bigcap v \mathbb{N}$ and suppose that $r_{s}\left(b_{1}\right)=r b_{1}$. There exists $c \in \Omega(s) \cap v \mathbb{N}$ such that both $b_{1}$ and $b_{2}$ divide $c$. In view of the above, $r_{s}\left(b_{1}\right)=r b_{1}$ implies $r_{s}(c)=r c$, which implies $r_{s}\left(b_{2}\right)=r b_{2}$. This completes the proof of the lemma.

Recall that for an infinite Steinitz number $s$ and a real number $r$, $1 \leqslant r<\infty$,

$$
\begin{aligned}
S(r, s) & =\left\{\frac{a}{b} s \mid a, b \in \mathbb{N} ; b \in \Omega(s), a \leqslant r b\right\}, \\
S^{+}(r, s) & =\left\{\frac{a}{b} s \mid a, b \in \mathbb{N} ; b \in \Omega(s), a<r b\right\} .
\end{aligned}
$$

If $r$ is an irrational number or $r=u / v$, the integers $u, v$ are coprime and $v \notin \Omega(s)$ then $S(r, s)=S^{+}(r, s)$. If $r=u / v, v \in \Omega(s)$ then $S^{+}(r, s) \varsubsetneqq S(r, s)$.

Lemma 6. The subsets $S(r, s)$ and $S^{+}(r, s)$ are saturated.

Proof. The condition 1) in the definition of saturated subsets is obviously satisfied. Let us check the condition 2). Let $(a / b) \cdot s \in S(r, s)$ (respectively, $\left.(a / b) \cdot s \in S^{+}(r, s)\right)$, where $a, b$ are coprime natural numbers, $b \in \Omega(s)$. Then $a \leqslant r b$ (respectively, $a<r b$ ). Suppose that $c \in \Omega\left(\frac{a}{b} s\right)$. We need to show that $(a \cdot s) /(b \cdot c) \in S(r, s)$ (respectively, $\left.(a \cdot s) /(b \cdot c) \in S^{+}(r, s)\right)$. Let $d=\operatorname{gcd}(a, c), a=d a^{\prime}, c=d c^{\prime}$. Then

$$
\frac{a s}{b c}=\frac{a^{\prime}}{b c^{\prime}} s \in \mathbb{S N} \text {. }
$$

Since the number $b c^{\prime}$ is coprime with $a^{\prime}$ it follows that $b c^{\prime} \in \Omega(s)$. The inequality $a^{\prime} \leqslant r b c^{\prime}$ (respectively, $a^{\prime}<r b c^{\prime}$ ) is equivalent to the inequality $a \leqslant r b c$ (respectively, $a<r b c$ ). The latter inequality follows from $a \leqslant r b$ (respectively, $a<r b$ ). The condition 2 ) is verified.

Let us check the condition 3). As above, we assume that $a, b$ are coprime natural numbers, $b \in \Omega(s)$ and $a / b \in S(r, s)$ (respectively, $a / b \in$ $\left.S^{+}(r, s)\right)$. Let $c \in \Omega((a / b) \cdot s), \operatorname{gcd}(a, c)=d, a=d a^{\prime}, c=d c^{\prime}$. We have shown above that $b c^{\prime} \in \Omega(s)$. Let $n \in \mathbb{N}$ and $n \cdot(a s /(b c)) \in S(r, s)$ 
(respectively, $\left.n \cdot(a s /(b c)) \in S^{+}(r, s)\right)$. Then $n a^{\prime} \leqslant r b c^{\prime}$ (respectively, $\left.n a^{\prime}<r b c^{\prime}\right)$. This immediately implies that for any $i, 1 \leqslant i \leqslant n$, we have $i a^{\prime} \leqslant r b c^{\prime}$ (respectively, $i a^{\prime}<r b c^{\prime}$ ). Hence, $i \cdot(a s / b) \in S(r, s)$ (respectively, $\left.i \cdot(a s / b) \in S^{+}(r, s)\right)$.

Lemma 7. Let $r=u / v$, where $u, v$ are coprime natural numbers. Let $s$ be an infinite Steinitz number and $v \in \Omega(s)$. Then the set $S^{+}(r, s)$ is not equal to any of the sets $S\left(r^{\prime}, s^{\prime}\right), r^{\prime} \in[1, \infty), s^{\prime} \in \mathbb{S}$.

Proof. Let $s_{2} \in S\left(r, s_{1}\right)$ (respectively, $s_{2} \in S^{+}\left(r, s_{1}\right)$ ). Then $s_{2}=(a / b) \cdot s_{1}$, where $a, b \in \mathbb{N}, b \in \Omega\left(s_{1}\right)$. By Lemma 4 ,

$$
S\left(r, s_{1}\right)=S\left(r \frac{b}{a}, s_{2}\right) \quad\left(\text { respectively, } S^{+}\left(r, s_{1}\right)=S^{+}\left(r \frac{b}{a}, s_{2}\right)\right) .
$$

We showed that the set $S(r, s)$ (respectively, $S^{+}(r, s)$ ) is determined by any Steinitz number $s^{\prime} \in S(r, s)$ (respectively, $s^{\prime} \in S^{+}(r, s)$ ) with an appropriate recalibration of $r$.

Let $S=S\left(r_{1}, s_{1}\right)=S^{+}\left(r_{2}, s_{2}\right)$. Choosing an arbitrary Steinitz number $s \in S$ we get $S\left(r_{1}^{\prime}, s\right)=S^{+}\left(r_{2}^{\prime}, s\right)$ for some $r_{1}^{\prime}, r_{2}^{\prime} \in[1, \infty)$. The number $r_{2}^{\prime}=u / v$ is rational, $\operatorname{gcd}(u, v)=1$ and $v \in \Omega(s)$.

The number $r$ is uniquely determined by a saturated subset $S$ and a choice of $s \in S$. Hence $r_{1}^{\prime}=r_{2}^{\prime}$. Now it remains to notice that for a rational number $r=u / v, \operatorname{gcd}(u, v)=1$, and an infinite Steinitz number $s$ such that $v \in \Omega(s)$ we have $S(r, s) \neq S^{+}(r, s)$. This completes the proof of the lemma.

Lemma 8. Let $S \subset \mathbb{S N} \backslash \mathbb{N}$ be a saturated subset of finite type, $s \in S$, $r=r_{S}(s) \in[1, \infty)$. Then $S=S(r, s)$ or $S=S^{+}(r, s)$.

Proof. Recall that for a natural number $b \in \Omega(s)$ we defined

$$
r_{s}(b)=\max \left\{i \in \mathbb{N} \mid i \frac{s}{b} \in S\right\} \text {. }
$$

We showed that if $r$ is an irrational number or $r=u / v$;,$v$ are coprime and $v \notin \Omega(s)$, then $r_{s}(b)=[r b]$ for an arbitrary $b \in \Omega(s)$.

An arbitrary Steinitz number $s^{\prime} \in S$ is representable as $s^{\prime}=(a / b) \cdot s$, where $a, b$ are coprime natural numbers. Clearly, $b \in \Omega(s)$ and $a \leqslant r_{s}(b)=$ $[r b]$. That is why in the case when $r$ is irrational or $r=u / v, \operatorname{gcd}(u, v)=1$, $v \notin \Omega(s)$, we have $S=S(r, s)=S^{+}(r, s)$.

Suppose now that $r=u / v, \operatorname{gcd}(u, v)=1, v \in \Omega(s)$. If $b \in \Omega(s) \backslash v \mathbb{N}$ then as above $r_{s}(b)=[r b]$. By Lemma 5, either for all $b \in \Omega(s) \cap v \mathbb{N}$ we have $r_{s}(b)=r b$ or for all $b \in \Omega(s) \cap v \mathbb{N}$ we have $r_{s}(b)=r b-1$. In the first case $S=S(r, s)$, in the second case $S=S^{+}(r, s)$. 
Lemma 9. Let $S \subseteq \mathbb{N}$ be a saturated subset. Then either $S=\{1,2, \ldots, n\}$ for some $n \in \mathbb{N}$ or $S=\mathbb{N}$.

Proof. First, notice that the subsets $\{1,2, \ldots, n\}$ and $\mathbb{N}$ are saturated.

Now let $S \subseteq \mathbb{N}$ be a saturated subset. If $n \in S$ then $n \in \Omega(n)$ and $n \cdot(n / n) \in S$. By the condition 3), all natural numbers $i=i \cdot(n / n)$, $1 \leqslant i \leqslant n$, lie in $S$. This implies the assertion of the lemma.

Now, Theorem 2 follows from Lemmas 8, 9 .

\section{Countable-dimensional locally matrix algebras}

For an algebra $A$ and an idempotent $0 \neq e \in A$ we call the subalgebra eAe a corner of the algebra $A$.

Let $A_{1} \subset A_{2} \subset \cdots$ be an ascending chain of unital locally matrix algebras, $A_{i}$ is a corner of the algebra $A_{i+1}, i \geqslant 1$,

$$
A=\bigcup_{i=1}^{\infty} A_{i}
$$

Clearly, $\operatorname{Spec}\left(A_{1}\right) \subseteq \operatorname{Spec}\left(A_{2}\right) \subseteq \cdots$.

Lemma 10.

$$
\operatorname{Spec}(A)=\bigcup_{i=1}^{\infty} \operatorname{Spec}\left(A_{i}\right)
$$

Proof. For an arbitrary idempotent $e \in A_{i}$ we have $e A_{i} e=e A e$, hence $\operatorname{Spec}\left(A_{i}\right) \subseteq \operatorname{Spec}(A)$. On the other hand, an arbitrary idempotent $e \in A$ lies in one of the subalgebras $A_{i}$. Hence $\mathbf{s t}(e A e)=\operatorname{st}\left(e A_{i} e\right) \in \operatorname{Spec}\left(A_{i}\right)$.

Proof of Theorem 3 (1). To start with we notice that $\{1,2, \ldots, n\}=$ $\operatorname{Spec}\left(M_{n}(\mathbb{F})\right)$. Let $s$ be a Steinitz number. In [2], we showed that there exists a unital locally matrix algebra $A, \operatorname{dim}_{\mathbb{F}} A \leqslant \aleph_{0}$, such that $\operatorname{st}(A)=s$. Consider the algebra $M_{\infty}(A)$ of infinite $\mathbb{N} \times \mathbb{N}$-matrices, having finitely many nonzero entries. The algebra $M_{n}(A)$ of $n \times n$-matrices over $A$ is embedded in $M_{\infty}(A)$ as a north-west corner,

$$
M_{1}(A) \subset M_{2}(A) \subset \cdots, \quad M_{\infty}(A)=\bigcup_{n=1}^{\infty} M_{n}(A) .
$$


In particular, it implies that $M_{\infty}(A)$ is a locally matrix algebra. We will show that

$$
\operatorname{Spec}\left(M_{\infty}(A)\right)=S(\infty, s) .
$$

Indeed, by Lemma 10,

$$
\operatorname{Spec}\left(M_{\infty}(A)\right)=\bigcup_{n=1}^{\infty} \operatorname{Spec}\left(M_{n}(A)\right) .
$$

We have $\mathbf{s t}\left(M_{n}(A)\right)=n s$. In [4], we showed that

$$
\operatorname{Spec}\left(M_{n}(A)\right)=\left\{\frac{a}{b} n s \mid b \in \Omega(n s), a, b \in \mathbb{N} ; 1 \leqslant a \leqslant b\right\} .
$$

This implies $\operatorname{Spec}\left(M_{n}(A)\right) \subseteq S(\infty, s)$. A Steinitz number $(a / b) \cdot s, b \in \Omega(s)$, lies in $\operatorname{Spec}\left(M_{n}(A)\right)$ provided that $a / b \leqslant n$. This completes the proof of (5). In particular, $\operatorname{Spec}\left(M_{\infty}(\mathbb{F})\right)=\mathbb{N}$.

Consider now a saturated subset $S=S(r, s)$ or $S=S^{+}(r, s), 1 \leqslant$ $r<\infty$, where $s$ is an infinite Steinitz number. Choose a sequence $b_{1}, b_{2}$, $\ldots \in \Omega(s)$ such that $b_{i}$ divides $b_{i+1}, i \geqslant 1$, and $s$ is the least common multiple of $b_{i}, i \geqslant 1$. There exists a unique (up to isomorphism) unital countable-dimensional locally matrix algebra $A_{s / b_{i}}$ such that $\mathbf{s t}\left(A_{s / b_{i}}\right)=$ $s / b_{i}$. Let $A_{i}=M_{r_{s}\left(b_{i}\right)}\left(A_{s / b_{i}}\right)$. We have

$$
\operatorname{st}\left(A_{s / b_{i}}\right)=\operatorname{st}\left(\left(M_{b_{i+1} / b_{i}}\left(A_{s / b_{i+1}}\right)\right)=s / b_{i+1} .\right.
$$

Hence, by Glimm's Theorem, $A_{s / b_{i}} \cong M_{b_{i+1} / b_{i}}\left(A_{s / b_{i+1}}\right)$ and, therefore,

$$
A_{i}=M_{r_{s}\left(b_{i}\right)}\left(A_{s / b_{i}}\right) \cong M_{r_{s}\left(b_{i}\right) \cdot \frac{b_{i+1}}{b_{i}}}\left(A_{s / b_{i+1}}\right) .
$$

By the inequality $(1), r_{s}\left(b_{i}\right) \cdot\left(b_{i+1} / b_{i}\right) \leqslant r_{s}\left(b_{i+1}\right)$. Hence, the algebra $A_{i}$ is embeddable in the algebra $A_{i+1}$ as a north-west corner. Let

$$
A=\bigcup_{i=1}^{\infty} A_{i}
$$

We will show that $\operatorname{Spec}(A)=S$. Let $0 \neq e \in A$ be an idempotent. Then $e \in A_{i}$ for some $i \geqslant 1$. In [4], we showed that

$$
\mathbf{s t}\left(e A_{i} e\right)=\frac{a}{b} \mathbf{s t}\left(A_{i}\right)
$$


where $a, b \in \mathbb{N} ; a, b$ are coprime natural numbers; $b \in \Omega\left(\mathbf{s t}\left(A_{i}\right)\right), a \leqslant b$. Furthermore,

$$
\mathbf{s t}\left(A_{i}\right)=r_{s}\left(b_{i}\right) \frac{s}{b_{i}}, \quad \mathbf{s t}\left(e A_{i} e\right)=\frac{a}{b} r_{s}\left(b_{i}\right) \frac{s}{b_{i}} .
$$

Let $d=\operatorname{gcd}\left(b, r_{s}\left(b_{i}\right)\right), b=d b^{\prime}, r_{s}\left(b_{i}\right)=d \cdot r_{s}\left(b_{i}\right)^{\prime}$. So,

$$
\mathbf{s t}\left(e A_{i} e\right)=\frac{a \cdot r_{s}\left(b_{i}\right)^{\prime}}{b^{\prime}} \cdot \frac{s}{b_{i}} \in \mathbb{S N} .
$$

This implies that $b^{\prime} \in \Omega\left(s / b_{i}\right)$. Therefore, $b^{\prime} b_{i} \in \Omega(s)$. To show that st $\left(e A_{i} e\right)$ lies in $S(r, s)$ (respectively, $S^{+}(r, s)$ ) we need to verify that $a \cdot r_{s}\left(b_{i}\right)^{\prime} \leqslant r b^{\prime} b_{i}$ (respectively, $\left.a \cdot r_{s}\left(b_{i}\right)^{\prime}<r b^{\prime} b_{i}\right)$. Multiplying both sides of the inequality by $d$ we get $a \cdot r_{s}\left(b_{i}\right) \leqslant r b b_{i}$ (respectively, $\left.a \cdot r_{s}\left(b_{i}\right)<r b b_{i}\right)$. This inequality holds since $a \leqslant b$ and $r_{s}\left(b_{i}\right) \leqslant r \cdot b_{i}$ (respectively, $a \leqslant b$ and $\left.r_{s}\left(b_{i}\right)<r \cdot b_{i}\right)$. We proved that $\operatorname{Spec}(A) \subseteq S$.

Let us show that $S \subseteq \operatorname{Spec}(A)$. Consider a Steinitz number $(a / b) \cdot s \in S$, where $a, b \in \mathbb{N} ; b \in \Omega(s), a \leqslant r b$ in the case $S=S(r, s)$ or $a<r b$ in the case $S=S^{+}(r, s)$.

There exists a member of our sequence $b_{i}$ such that $b$ divides $b_{i}$, $b_{i}=k \cdot b, k \in \mathbb{N}$. Then $(a / b) \cdot s=\left(a k / b_{i}\right) \cdot s$.

We will show that $a k \leqslant r_{s}\left(b_{i}\right)$. Indeed, multiplying both sides of the inequality by $b$ we get $a b_{i} \leqslant r_{s}\left(b_{i}\right) b$. Let $S=S(r, s)$. Then $a \leqslant r b$. Since $a \in \mathbb{N}$ it implies $a \leqslant[r b]$. Furthermore, $r_{s}\left(b_{i}\right)=\left[r b_{i}\right]=[r b k]$. So, it is sufficient to show that $[r b] k \leqslant[r b k]$. This inequality holds since $[r b] k$ is an integer and $[r b] k \leqslant r b k$.

Now suppose that $S=S^{+}(r, s)$. Then $a<r b$,

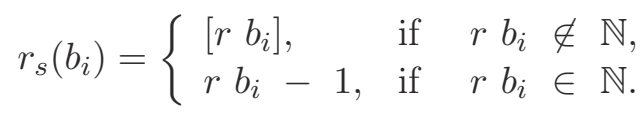

There are three possibilities:

1) $r b \in \mathbb{N}$ and, therefore, $r b_{i} \in \mathbb{N}$. In this case $a \leqslant r b-1, r_{s}\left(b_{i}\right)=$ $r b_{i}-1$. We have $a b_{i} \leqslant(r b-1) b_{i} \leqslant\left(r b_{i}-1\right) b=r_{s}\left(b_{i}\right) b$;

2) $r b \notin \mathbb{N}$, but $r b_{i} \in \mathbb{N}$. In this case $a \leqslant[r b], r_{s}\left(b_{i}\right)=r b_{i}-1$, we have $a b_{i} \leqslant[r b] b_{i}, r_{s}\left(b_{i}\right) b=\left(r b_{i}-1\right) b$. Hence, it is sufficient to show that $[r b] k \leqslant r b_{i}-1=r b k-1$. The number $[r b] k$ is an integer and $[r b] k<r b k$ since $[r b]<r b$. This implies the claimed inequality;

3) $r b_{i} \notin \mathbb{N}$ and, therefore, $r b \notin \mathbb{N}$. In this case $a b_{i} \leqslant[r b] b k, r_{s}\left(b_{i}\right) b=$ $[r b k] b$ and it remains to notice that $[r b] k \leqslant[r b k]$. 
We showed that both for $S=S(r, s)$ and for $S=S^{+}(r, s)$ there holds the inequality $a k \leqslant r_{s}\left(b_{i}\right)$.

Recall that $A_{i}=M_{r_{s}\left(b_{i}\right)}\left(A_{s / b_{i}}\right)$. Consider the north-east corner $M_{a k}\left(A_{s / b_{i}}\right)$ of the algebra $A_{i}$. We have

$$
\text { st }\left(M_{a k}\left(A_{s / b_{i}}\right)\right)=a k \cdot \frac{s}{b_{i}}=\frac{a}{b} s,
$$

and, therefore, $S \subseteq \operatorname{Spec}(A)$. This completes the proof of Theorem 3 (1).

For the proof of Theorem 3 (2) we will need several lemmas on extensions of isomorphisms.

Lemma 11. Let $A$ be a locally matrix algebra and let $A_{1}$ be a subalgebra of $A$ such that $A_{1} \cong M_{n}(\mathbb{F})$. Then every automorphism of the algebra $A_{1}$ extends to an automorphism of the algebra $A$.

Proof. Let $e$ be the identity element of the subalgebra $A_{1}$. Then the corner $e A e$ is a unital locally matrix algebra. Let $C$ be the centralizer of the subalgebra $A_{1}$ in $e A e$. By Wedderbern's Theorem (see [6, 8]), we have $e A e=A_{1} \otimes_{\mathbb{F}} C$. An arbitrary automorphism $\varphi$ of the subalgebra $A_{1}$ is inner, that is, there exists an invertible element $x$ of the subalgebra $A_{1}$ such that $\varphi(a)=x^{-1} a x$ for all elements $a \in A_{1}$. The conjugation by the element $x \otimes e$ extends $\varphi$ to an automorphism of the algebra $e A e$. Consider the Peirce decomposition

$$
A=e A e+e A(1-e)+(1-e) A e+(1-e) A(1-e),
$$

and the mapping

$\tilde{\varphi}: A \ni a \mapsto x^{-1} a x+x^{-1} a(1-e)+(1-e) a x+(1-e) a(1-e)$.

The mapping $\tilde{\varphi}$ extends $\varphi$ and $\tilde{\varphi} \in \operatorname{Aut}(A)$. This completes the proof of the lemma.

Lemma 12. Let $A$ be a unital locally matrix algebra with an idempotent $e \neq 0$. Then an arbitrary automorphism of the corner eAe extends to an automorphism of the algebra $A$.

Proof. Suppose at first that an automorphism $\varphi$ of the algebra $e A e$ is inner, and there exists an element $x_{e} \in e A e$ that is invertible in the algebra $e A e$ such that $\varphi(a)=x_{e}^{-1} a x_{e}$ for an arbitrary element $a \in e A e$. The 
element $x=x_{e}+(1-e)$ is invertible in the algebra $A$. So, conjugation by the element $x$ extends $\varphi$.

Now let $\varphi$ be an arbitrary automorphism of the corner $e A e$. Let $A_{1} \subseteq A$ be a subalgebra such that $1, e \in A_{1}$ and $A_{1} \cong M_{m}(\mathbb{F})$ for some $m \geqslant 1$. Consider $A_{2} \subseteq A$ such that $A_{1} \subseteq A_{2}, \varphi\left(e A_{1} e\right) \subseteq A_{2}$ and $A_{2} \cong M_{n}(\mathbb{F})$ for some $n \geqslant 1$. Consider the embedding

$$
\varphi: e A_{1} e \rightarrow \varphi\left(e A_{1} e\right) \subseteq e A_{2} e
$$

that preserves the identity element $e$. By Skolem-Noether Theorem (see [6]), there exists an invertible element $x_{e} \in e A_{2} e$ such that $\varphi(a)=x_{e}^{-1} a x_{e}$ for an arbitrary element $a \in e A_{1} e$.

As noticed above, there exists an automorphism $\psi$ of the algebra $A$ that extends the automorphism $e A e \rightarrow e A e, a \mapsto x_{e}^{-1} a x_{e}$. The composition $\psi^{-1} \circ \varphi$ leaves all elements of the algebra $e A_{1} e$ fixed. Since it is sufficient to prove that the automorphism $\psi^{-1} \circ \varphi \in \operatorname{Aut}(e A e)$ extends to an automorphism of $A$ we will assume without loss of generality that the automorphism $\varphi \in \operatorname{Aut}(e A e)$ fixes all elements of $e A_{1} e$.

Let $C$ be the centralizer of the subalgebra $A_{1}$ in $A$. Then $A=A_{1} \otimes_{\mathbb{F}} C$ and $e A e=e A_{1} e \otimes_{\mathbb{F}} C$. Since the subalgebra $e \otimes_{\mathbb{F}} C$ is the centralizer of $e A_{1} e \otimes_{\mathbb{F}} C$ in the algebra $e A e$ it follows that $e \otimes_{\mathbb{F}} C$ is invariant with respect to the automorphism $\varphi$. Hence, there exists an automorphism $\theta \in \operatorname{Aut}(C)$ such that $\varphi(a \otimes c)=a \otimes \theta(c)$ for all elements $a \in e A e, c \in C$. So, the automorphism $\tilde{\varphi}(a \otimes c)=a \otimes \theta(c), a \in A_{1}, c \in C$, extends $\varphi$. This completes the proof of the lemma.

Lemma 13. Let $A$ be a unital locally matrix algebra with nonzero idempotents $e_{1}, e_{2}$. An arbitrary isomorphism $\varphi: e_{1} A e_{1} \rightarrow e_{2} A e_{2}$ extends to an automorphism of the algebra $A$.

Proof. There exists a subalgebra $A_{1} \subseteq A$ such that $1, e_{1}, e_{2} \in A_{1}$ and $A_{1} \cong M_{n}(\mathbb{F})$ for some $n \geqslant 1$. Let $r_{i}$ be the matrix range of the idempotent $e_{i}$ in $A_{1}, i=1,2$. In [4], it was shown that

$$
\mathbf{s t}\left(e_{1} A e_{1}\right)=\frac{r_{1}}{n} \cdot \mathbf{s t}(A), \quad \mathbf{s t}\left(e_{2} A e_{2}\right)=\frac{r_{2}}{n} \cdot \mathbf{s t}(A) .
$$

Since $e_{1} A e_{1} \cong e_{2} A e_{2}$ it follows that $r_{1}=r_{2}$. In the matrix algebra $M_{n}(\mathbb{F})$ any two idempotents of the same range are conjugate via an automorphism. Hence, the idempotents $e_{1}, e_{2}$ are conjugate via an automorphism of $A_{1}$. By Lemma 11, an arbitrary automorphism of $A_{1}$ extends to an automorphism of the algebra $A$. Now the assertion of the lemma follows from Lemma 12. 
Lemma 14. Let $A, B$ be isomorphic unital locally matrix algebras with nonzero idempotents e $\in A, f \in B$. An arbitrary isomorphism eAe $\rightarrow f B f$ extends to an isomorphism $A \rightarrow B$.

Proof. Let $\varphi: A \rightarrow B, \psi: e A e \rightarrow f B f$ be isomorphisms. Then

$$
\varphi^{-1} \circ \psi: e A e \rightarrow \varphi^{-1}(f) A \varphi^{-1}(f)
$$

is an isomorphism of two corners of the algebra $A$. By Lemma 13, $\varphi^{-1} \circ \psi$ extends to an automorphism $\chi$ of the algebra $A$, the isomorphism $\varphi \circ \chi$ extends $\psi$.

Lemma 15. Let $A$ be a unital locally matrix algebra and let $s_{1}, s_{2}$ be Steinitz numbers from $\operatorname{Spec}(A)$. Suppose that $s_{2} / s_{1}>1$. Let $e_{1} \in A$ be an idempotent such that $\mathbf{s t}\left(e_{1} A e_{1}\right)=s_{1}$. Then there exists an idempotent $e_{2}>e_{1}$ such that $\mathbf{s t}\left(e_{2} A e_{2}\right)=s_{2}$.

Proof. Since $s_{2} \in \operatorname{Spec}(A)$ there exists an idempotent $e^{\prime} \in A$ such that $\operatorname{st}\left(e^{\prime} A e^{\prime}\right)=s_{2}$. Choose a subalgebra $A_{1} \subseteq A$ such that $e_{1}, e^{\prime} \in A_{1}$ and $A_{1} \cong M_{n}(\mathbb{F})$.

Let $r_{1}, r_{2}$ be the matrix ranges of $e_{1}, e^{\prime}$ in $M_{n}(\mathbb{F})$, respectively. In [4], it was shown that

$$
\mathbf{s t}\left(e_{1} A e_{1}\right)=s_{1}=\frac{r_{1}}{n} \mathbf{s t}(A), \quad \mathbf{s t}\left(e^{\prime} A e^{\prime}\right)=s_{2}=\frac{r_{2}}{n} \mathbf{s t}(A) .
$$

Hence $r_{2}>r_{1}$. Since every idempotent in the algebra $M_{n}(\mathbb{F})$ is diagonalizable there exist automorphisms $\varphi, \psi$ of the algebra $A_{1}$ such that $\psi\left(e^{\prime}\right)>\varphi\left(e_{1}\right)$. By Lemma 11, the automorphisms $\varphi, \psi$ extend to automorphisms $\widetilde{\varphi}, \widetilde{\psi}$ of the algebra $A$, respectively.

Let $e_{2}=\widetilde{\varphi}^{-1}\left(\psi\left(e^{\prime}\right)\right)$. Then $e_{2}>e_{1}$ and $\mathbf{s t}\left(e_{2} A e_{2}\right)=s_{2}$, which completes the proof of the lemma.

Proof of Theorem 3 (2). Let $A, B$ be countable-dimensional locally matrix algebras, $\operatorname{Spec}(A)=\operatorname{Spec}(B)$. Choose bases $a_{1}, a_{2}, \ldots$ and $b_{1}, b_{2}, \ldots$ in the algebras $A, B$, respectively.

We will construct ascending chains of corners $\{0\}=A_{0} \subset A_{1} \subset A_{2} \subset$ $\ldots$ in the algebra $A$ and $\{0\}=B_{0} \subset B_{1} \subset B_{2} \subset \cdots$ in the algebra $B$, such that

$$
\bigcup_{i=0}^{\infty} A_{i}=A, \quad \bigcup_{i=0}^{\infty} B_{i}=B
$$

and $a_{1}, \ldots, a_{i} \in A_{i}, b_{1}, \ldots, b_{i} \in B_{i}, \mathbf{s t}\left(A_{i}\right)=\mathbf{s t}\left(B_{i}\right)$ for all $i \geqslant 1$. 
Suppose that corners $\{0\}=A_{0} \subset A_{1} \subset A_{2} \subset \cdots \subset A_{n},\{0\}=B_{0} \subset$ $B_{1} \subset B_{2} \subset \cdots \subset B_{n}$ have already been selected, $n \geqslant 0$. There exist corners $A^{\prime} \subset A, B^{\prime} \subset B$ in the algebras $A, B$, respectively, such that $A_{n} \subset A^{\prime}, a_{n+1} \in A^{\prime}$ and $B_{n} \subset B^{\prime}, b_{n+1} \in B^{\prime}$. The Steinitz numbers $\mathbf{s t}\left(A^{\prime}\right), \mathbf{s t}\left(B^{\prime}\right)$ lie in the same saturated subset of $\mathbb{S N}$, therefore, they are rationally connected.

Suppose that $\mathbf{s t}\left(B^{\prime}\right) \geqslant \operatorname{st}\left(A^{\prime}\right)$. Let $=e^{\prime}$ be an idempotent of the algebra $A$ such that $A^{\prime}=e^{\prime} A e^{\prime}$. The Steinitz number st $\left(B^{\prime}\right)$ lies in $\operatorname{Spec}(A)$. Hence, by Lemma 15 , there exists an idempotent $e \in A$ such that $e \geqslant e^{\prime}$ and $\mathbf{s t}(e A e)=\operatorname{st}\left(B^{\prime}\right)$. Choose $A_{n+1}=e A e, B_{n+1}=B^{\prime}$. The chains $\{0\}=A_{0} \subset A_{1} \subset A_{2} \subset \cdots$ and $\{0\}=B_{0} \subset B_{1} \subset B_{2} \subset \cdots$ have been constructed.

By Lemma 14, every isomorphism $A_{i} \rightarrow B_{i}$ extends to an isomorphism $A_{i+1} \rightarrow B_{i+1}$. This gives rise to a sequence of isomorphisms $\varphi_{i}: A_{i} \rightarrow B_{i}$, $i \geqslant 0$, where each $\varphi_{i+1}$ extends $\varphi_{i}$. Taking the union $\cup_{i \geqslant 0} \varphi_{i}$ we get an isomorphism from the algebra $A$ to the algebra $B$. This completes the proof of the theorem.

Proof of Theorem 4. It is easy to see that a locally matrix algebra $A$ is a unital if and only if the set of idempotents of $A$ has a largest element: an identity. This is equivalent to $\operatorname{Spec}(A)$ containing a largest Steinitz number. Among saturated sets of Steinitz numbers only $\{1,2, \ldots, n\}$ and $S(r, s)$, $s \in \mathbb{S N} \backslash \mathbb{N}, r=u / v ; u$ and $v$ are coprime natural numbers, $v \in \Omega(s)$, satisfy this assumption.

\section{Embeddings of locally matrix algebras}

Lemma 16. Let $S_{1}, S_{2}$ be saturated sets of Steinitz numbers. Then either $S_{1} \cap S_{2}=\varnothing$ or one of the sets $S_{1}, S_{2}$ contains the other one.

Proof. Let $s \in S_{1} \cap S_{2}$. If $s \in \mathbb{N}$ then, by Lemma 9, each set $S_{i}$ is either a segment $[1, n], n \geqslant 1$, or the whole $\mathbb{N}$. In this case the assertion of the lemma is obvious.

Suppose that the number $s$ is infinite. Then by Theorem 2, $S_{i}=S\left(r_{i}, s\right)$ or $S_{i}=S^{+}\left(r_{i}, s\right)$, where $r_{i}=r_{S_{i}}(s) \in[1, \infty) \cup\{\infty\}, i=1,2$. Clearly, if $r_{S_{1}}(s)<r_{S_{2}}(s)$ then $S_{1} \varsubsetneqq S_{2}$. If $r_{S_{1}}(s)=r_{S_{2}}(s)$ then

$$
S_{1}, S_{2}=\left[\begin{array}{l}
S(r, s) \\
S^{+}(r, s) \\
S(\infty, s)
\end{array}\right.
$$


and $S^{+}(r, s) \subseteq S(r, s) \subset S(\infty, s)$ for any $r \in[1, \infty)$. This completes the proof of the lemma.

Let $A$ be a locally matrix algebra. A subalgebra $B \subseteq A$ is called an approximative corner of $A$ if $B$ is the union of an increasing chain of corners. In other words, there exist idempotents $e_{0}, e_{1}, e_{2}, \ldots$ such that

$$
e_{0} A e_{0} \subseteq e_{1} A e_{1} \subseteq e_{2} A e_{2} \subseteq \cdots, \quad B=\bigcup_{i=0}^{\infty} e_{i} A e_{i} .
$$

It is easy to see that an approximative corner of a locally matrix algebra is a locally matrix algebra.

Theorem 5. Let $A, B$ be countable-dimensional locally matrix algebras. Then $B$ is embeddable in $A$ as an approximative corner if and only if $\operatorname{Spec}(B) \subseteq \operatorname{Spec}(A)$.

Proof. If $B$ is an approximative corner of $A$ then every corner of $B$ is a corner of $A$, hence $\operatorname{Spec}(B) \subseteq \operatorname{Spec}(A)$.

Suppose now that $\operatorname{Spec}(B) \subseteq \operatorname{Spec}(A)$. If the algebra $B$ is unital then it embedds in the algebra $A$ as a corner. Indeed, the embedding $\operatorname{Spec}(B) \subseteq \operatorname{Spec}(A)$ implies that there exists an idempotent $e \in A$ such that $\mathbf{s t}(B)=\mathbf{s t}(e A e)$. By Glimm's Theorem [7], we have $B \cong e A e$.

Suppose now that the algebra $B$ is not unital. Then there exists a sequence of idempotents $0=f_{0}, f_{1}, f_{2}, \ldots$ of algebra $B$ such that

$$
\{0\}=f_{0} B f_{0} \varsubsetneqq f_{1} B f_{1} \varsubsetneqq f_{2} B f_{2} \varsubsetneqq \cdots, \bigcup_{i=0}^{\infty} f_{i} B f_{i}=B .
$$

We will construct a sequence of idempotents $e_{0}, e_{1}, e_{2}, \ldots$ in the algebra $A$ such that

$$
e_{0} A e_{0} \varsubsetneqq e_{1} A e_{1} \varsubsetneqq e_{2} A e_{2} \varsubsetneqq \cdots, \quad \operatorname{st}\left(f_{i} B f_{i}\right)=\operatorname{st}\left(e_{i} A e_{i}\right)
$$

for an arbitrary $i \geqslant 0$. Let $e_{0}=0$. Suppose that we have already selected idempotents $e_{0}, e_{1}, \ldots, e_{n} \in A$ such that $e_{0} A e_{0} \subset e_{1} A e_{1} \subset \cdots \subset e_{n} A e_{n}$ and $\mathbf{s t}\left(e_{i} A e_{i}\right)=\mathbf{s t}\left(f_{i} B f_{i}\right), 0 \leqslant i \leqslant n$. We have

$$
\mathbf{s t}\left(f_{n+1} B f_{n+1}\right)>\operatorname{st}\left(f_{n} B f_{n}\right)=\operatorname{st}\left(e_{n} A e_{n}\right)
$$

and $\mathbf{s t}\left(f_{n+1} B f_{n+1}\right) \in \operatorname{Spec}(A)$. By Lemma 15, there exists an idempotent $e_{n+1} \in A$ such that $e_{n} A e_{n} \subset e_{n+1} A e_{n+1}$ and $\operatorname{st}\left(e_{n+1} A e_{n+1}\right)=$ $\mathbf{s t}\left(f_{n+1} B f_{n+1}\right)$, which proves existence of a sequence $e_{0}, e_{1}, e_{2}, \ldots$ 
The union

$$
A^{\prime}=\bigcup_{i=0}^{\infty} e_{i} A e_{i}
$$

is an approximative corner of the algebra $A$. By Glimm's Theorem [7], $e_{i} A e_{i} \cong f_{i} B f_{i}, i \geqslant 1$. By Lemma 10,

$$
\operatorname{Spec}\left(A^{\prime}\right)=\bigcup_{i=1}^{\infty} \operatorname{Spec}\left(e_{i} A e_{i}\right) \text { and } \operatorname{Spec}(B)=\bigcup_{i=1}^{\infty} \operatorname{Spec}\left(f_{i} B f_{i}\right) \text {. }
$$

Hence $\operatorname{Spec}(B)=\operatorname{Spec}\left(A^{\prime}\right)$. By Theorem $3(2)$, we have $A^{\prime} \cong B$, which completes the proof of the theorem.

\section{References}

[1] A. A. Baranov, Classification of the direct limits of involution simple associative algebras and the corresponding dimension groups, Journal of Algebra, 381, 2013, pp.73-95.

[2] O. Bezushchak and B. Oliynyk, Unital locally matrix algebras and Steinitz numbers, J. Algebra Appl., 19(9), 2020, Doi:10.1142/SO219498820501807.

[3] O. Bezushchak and B. Oliynyk, Primary decompositions of unital locally matrix algebras, Bull. Math. Sci., 10(1), 2020, Doi:10.1142/S166436072050006X.

[4] O. Bezushchak and B. Oliynyk, Morita equivalent unital locally matrix algebras, Algebra Discrete Math., 29(2), 2020, pp.173-179.

[5] J. Dixmier, On some $C^{*}$-algebras considered by Glimm, J. Funct. Anal., 1, 1967, pp.182-203.

[6] Yu. A. Drozd, V. V. Kirichenko, Finite dimensional algebras, Springer-Verlag, Berlin-Heidelberg-New York, 1994.

[7] J. G. Glimm, On a certain class of operator algebras, Trans. Amer. Math. Soc., 95(2), 1960, pp.318-340.

[8] N. Jacobson, Structure of rings, Colloquium Publications, 37, 1956.

[9] V. M. Kurochkin, On the theory of locally simple and locally normal algebras, Mat. Sb., Nov. Ser., 22(64)(3), 1948, pp.443-454.

[10] A. Kurosh, Direct decompositions of simple rings, Rec. Math. [Mat. Sbornik] N.S., 11(53)(3), 1942, pp. 245-264.

[11] E. Steinitz, Algebraische Theorie der Körper, Journal für die reine und angewandte Mathematik 137, 1910, pp.167-309.

\section{CONTACT INFORMATION}

Oksana Bezushchak Faculty of Mechanics and Mathematics, Taras Shevchenko National University of Kyiv, Volodymyrska, 60, Kyiv 01033, Ukraine E-Mail(s): mechmatknubezushchak@gmail.com

Received by the editors: 08.12.2020

and in final form 15.02.2021. 